\begin{tabular}{|c|c|c|}
\hline & Int.J.Curr.Microbiol.App.Sci (2021) 10(09): 538-544 & \\
\hline & $\begin{array}{l}\text { International Journal of Current Microbiology and Applied Sciences } \\
\text { ISSN: 2319-7706 Volume } \mathbf{1 0} \text { Number } 09(\mathbf{2 0 2 1 )} \\
\text { Journal homepage: } \underline{\text { http://www.ijcmas.com }}\end{array}$ & $\$ 9$ \\
\hline $\begin{array}{l}\text { EXCELLENT } \\
\text { PUBLISHERS }\end{array}$ & & \\
\hline
\end{tabular}

Original Research Article

https://doi.org/10.20546/ijcmas.2021.1009.062

\title{
Evaluation of Yield Loss Assessment Caused due to Curvularia Leaf Spot (Curvularia lunata) in Maize
}

\author{
Tarun Kumar Jatwa*, S. S. Sharma, Neeraj K. Meena, Irfan Khan, \\ Roop Singh and Indu
}

Department of Plant Pathology, Rajasthan Collage of Agriculture, MPUAT, Udaipur, Rajasthan

*Corresponding author

\begin{abstract}
A B S T R A C T
Keywords

Yield loss,

Curvularia lead

spot, Loss

assessment

Article Info

Accepted:

20 August 2021

Available Online:

10 September 2021

An experiment was conducted during Kahrif 2017 and Kharif 2018 at department of plant pathology, Rajasthan collage of Agriculture, Udaipur, Raj. to find out the average yield loss assessment caused due to Curvularia leaf spot of maize. During Kharif 2017 estimated average yield loss was $21.69 \%$ with $18.56 \%$ PDI and $1032.33 \mathrm{~kg} / \mathrm{plot}$ yield in protected plot similarly in Kharif 2018 yield loss was $22.49 \%$ and $992.73 \mathrm{~kg} /$ plot yield with $18.07 \%$ PDI. On average yield loss caused due to CLS in maize in both years was estimated $22.06 \%$.

\section{Introduction}

Maize (Zea mays L.), belonging to the family Gramineae is one of the important cereal crops of the world. The maize kernel, like that of other cereal grains, includes pericarp (6\%), endosperm $(82 \%)$ and germ $(12 \%)$. The main structural component of the endosperm is starch, a complex carbohydrate that constitutes on an average 71 per cent of the grain and is a source of concentrated energy. Several million people, particularly in the developing countries, derive their protein and calorie requirements from maize. The maize grain accounts for about 15 to 56 per cent of the total daily calories in diets of people in about

25 developing countries, particularly in Africa and Latin America, where animal protein is scarce and expensive and consequently, unavailable to a vast sector of the population (Prasanna et al., 2001). Maize is currently produced on nearly 100 million hectares in 125 developing countries and is among the three most widely in grown crops in 75 of those countries (Anonymous, 2012). These include seedling blights, stalk rots, foliar diseases, downy mildews and ear rots. Among the fungal diseases Curvularia lunata (Cochliobols lunatus) was recorded on maize by Curvularia leaf spot is potentially an important foliar disease in areas where the temperatures drop at night while the humidity
\end{abstract}


is high. The disease is known to affect maize from seedling stage till harvest. Loss in grain yield will be more if it occurs at flowering, silking and grain filling stages. Curvularia is a hyphomycete (mold) fungus which is a facultative pathogen of many plant species and of the soil. Conidia develop at the tips and sides of the spores and have a smooth texture. C. lunata is differentiated from other Curvularia species by its 3 septa and 4 cells, with the first and last cell usually of a paler shade of brown than those in the middle.

Conidia range from 9-15 $\mu \mathrm{m}$ in diameter and have a curved appearance (Macri and Dilenna, 1974). Importance of maize, it is being plagued by an array of diseases which include the leaf spot of maize, caused by Curvularia lunata. This disease is a very important seed and soil borne prevalent in the hot, humid maize areas. The disease produces or chlorotic spot with a light colored halo lesions are about $0.5 \mathrm{~cm}$ per spot when fully developed and this cause significant damage to maize up to 60 per cent due to great loss of photosynthetic region of the crop (Akinbode, 2010).

\section{Materials and Methods}

To know the extent of losses in yield due to CLS, a model suggested by Le Clerg, 1957 was followed. In this model it is mandatory to take minimum of 10 replications of protected and unprotected treatments. It is also necessary to inoculate all the replication of both treatments artificially to maintain homogenous disease pressure.

The experiment was taken in the field allotted to AICRP- Maize Pathology viz. B3A. The maize cultivar 'Surya' was sown in RBD in field during Kharif 2017 and Kharif 2018. The row length was $3 \mathrm{~m}$ plant to plant and row to row $30 \& 60 \mathrm{~cm}$ respectively. The crop was inoculated through spray and inoculation using conidial suspension on 40 DAS plants twice with two days interval. Inoculation was done in warm and humid condition at 5-6 pm before sunset.

The yield data in protected and unprotected were recorded replication wise and the reduction in PDI was also calculated. PDI and yield loss Cramer (1967) was calculated by adopting following formulas:

$$
\begin{aligned}
& \text { Sum of all individual } \\
& \begin{array}{lcc}
\text { Per cent } \\
\text { index }=
\end{array} \quad \text { disease } \frac{\text { disease ratings }}{\begin{array}{c}
\text { Total numbers of plants } \\
\text { assessed X Maximum rating }
\end{array}} \times 100 \\
& \text { Difference in yield of protected and } \\
& \text { Loss }=\frac{\text { Inoculated plot }}{\text { Yield in protected plot }} \times 100
\end{aligned}
$$

\section{Results and Discussion}

Loss assessment is pre requisite task to know how much disease cause loss in main crops during favourable condition. A paired $\mathrm{T}$ technique was used to find avoidable lose caused by CLS. We select the susceptible cultivar i.e. Surya for Curvularia leaf spot of maize. In paired $\mathrm{T}$ technique two plots were maintained one is protected plot and other is unprotected to know lose.

In both plots protected and unprotected was inoculated with mass multiplied pathogen culture and generated the artificial epiphytotic condition. Protected plot was sprayed with propiconazole at $0.2 \%$ twice in 15 days interval of time and unprotected plot was remain unsprayed with fungicide. Total 30 lines (15 replication) for both plots were maintained at $30 \times 60 \mathrm{~cm}$ distance. 
Table.1 Losses assessment of CLS of maize during Kharif 2017

\begin{tabular}{|c|c|c|c|c|c|}
\hline Replication & Treatment & $\begin{array}{c}\text { Disease } \\
\text { rating }\end{array}$ & PDI & $\begin{array}{c}\text { Yield } \\
\text { (gm/plot) }\end{array}$ & Yield loss (\%) \\
\hline \multirow[t]{2}{*}{ R1 } & Protected & 3 & $\begin{array}{c}22.5 \\
(28.32)\end{array}$ & 895 & \multirow[t]{2}{*}{23.57} \\
\hline & Unprotected & 7.5 & $\begin{array}{c}72.0 \\
(58.05)\end{array}$ & 684 & \\
\hline \multirow[t]{2}{*}{$\mathbf{R 2}$} & Protected & 3 & $\begin{array}{c}17.5 \\
(24.73)\end{array}$ & 985 & \multirow[t]{2}{*}{22.13} \\
\hline & Unprotected & 6.5 & $\begin{array}{c}74.16 \\
(59.45)\end{array}$ & 767 & \\
\hline \multirow[t]{2}{*}{ R3 } & Protected & 2.5 & $\begin{array}{c}20.0 \\
(26.57)\end{array}$ & 998 & \multirow[t]{2}{*}{18.63} \\
\hline & Unprotected & 6 & $\begin{array}{c}75.71 \\
(60.47)\end{array}$ & 812 & \\
\hline \multirow[t]{2}{*}{ R4 } & Protected & 2 & $\begin{array}{c}15.0 \\
(22.79)\end{array}$ & 1231 & \multirow[t]{2}{*}{22.17} \\
\hline & Unprotected & 7 & $\begin{array}{c}78.33 \\
(62.26)\end{array}$ & 958 & \\
\hline \multirow[t]{2}{*}{ R5 } & Protected & 3 & $\begin{array}{c}21.66 \\
(27.74)\end{array}$ & 910 & \multirow[t]{2}{*}{21.53} \\
\hline & Unprotected & 8 & $\begin{array}{c}72.85 \\
(58.60)\end{array}$ & 714 & \\
\hline \multirow[t]{2}{*}{ R6 } & Protected & 2.5 & $\begin{array}{c}16.00 \\
(23.58)\end{array}$ & 1037 & \multirow[t]{2}{*}{20.63} \\
\hline & Unprotected & 7 & $\begin{array}{c}67.33 \\
(55.14)\end{array}$ & 823 & \\
\hline \multirow[t]{2}{*}{ R7 } & Protected & 2 & $\begin{array}{c}14.28 \\
(22.20)\end{array}$ & 984 & \multirow[t]{2}{*}{24.59} \\
\hline & Unprotected & 7.5 & $\begin{array}{c}65.71 \\
(54.16) \\
\end{array}$ & 742 & \\
\hline \multirow[t]{2}{*}{ R8 } & Protected & 2.5 & $\begin{array}{c}18.57 \\
(25.75)\end{array}$ & 1072 & \multirow[t]{2}{*}{23.04} \\
\hline & Unprotected & 6.5 & $\begin{array}{c}70.0 \\
(56.79)\end{array}$ & 825 & \\
\hline \multirow[t]{2}{*}{ R9 } & Protected & 2 & $\begin{array}{c}16.00 \\
(23.58)\end{array}$ & 1124 & \multirow[t]{2}{*}{19.48} \\
\hline & Unprotected & 6 & $\begin{array}{c}64.28 \\
(53.30)\end{array}$ & 905 & \\
\hline \multirow[t]{2}{*}{ R10 } & Protected & 2.5 & $\begin{array}{c}20.0 \\
(26.57)\end{array}$ & 940 & \multirow[t]{2}{*}{23.29} \\
\hline & Unprotected & 6 & $\begin{array}{c}71.25 \\
(57.58)\end{array}$ & 721 & \\
\hline \multirow[t]{2}{*}{ R11 } & Protected & 2.5 & $\begin{array}{c}16.66 \\
(24.09)\end{array}$ & 1242 & \multirow[t]{2}{*}{25.76} \\
\hline & Unprotected & 7.5 & $\begin{array}{c}68.57 \\
(55.90)\end{array}$ & 922 & \\
\hline
\end{tabular}




\begin{tabular}{|c|c|c|c|c|c|}
\hline \multirow[t]{2}{*}{ R12 } & Protected & 2 & $\begin{array}{c}14.28 \\
(22.20)\end{array}$ & 1026 & \multirow[t]{2}{*}{19.98} \\
\hline & Unprotected & 6.5 & $\begin{array}{c}71.42 \\
(57.68)\end{array}$ & 821 & \\
\hline \multirow[t]{2}{*}{ R13 } & Protected & 2 & $\begin{array}{c}25.0 \\
(30.00)\end{array}$ & 964 & \multirow[t]{2}{*}{23.75} \\
\hline & Unprotected & 6.5 & $\begin{array}{c}74.44 \\
(59.63)\end{array}$ & 735 & \\
\hline \multirow[t]{2}{*}{ R14 } & Protected & 1.5 & $\begin{array}{c}16.66 \\
(24.09)\end{array}$ & 1167 & \multirow[t]{2}{*}{19.28} \\
\hline & Unprotected & 6 & $\begin{array}{c}71.42 \\
(57.68)\end{array}$ & 942 & \\
\hline \multirow[t]{3}{*}{ R15 } & Protected & 2 & 24.0 & 955 & \multirow[t]{3}{*}{17.59} \\
\hline & & & (29.33) & & \\
\hline & Unprotected & 6 & $\begin{array}{c}77.77 \\
(61.87)\end{array}$ & 787 & \\
\hline \multirow[t]{2}{*}{ Mean } & Protected & 2.3 & $\begin{array}{c}18.56 \\
(24.43)\end{array}$ & 1032.33 & \multirow{4}{*}{21.69} \\
\hline & Unprotected & 6.7 & $\begin{array}{c}71.68 \\
(57.90)\end{array}$ & 795.40 & \\
\hline \multicolumn{2}{|c|}{$T$ calculated } & 27.66 & $\begin{array}{c}52.92 \\
(47.70)\end{array}$ & 27.34 & \\
\hline \multicolumn{2}{|c|}{ T tabulated } & 12.70 & 12.70 & 12.70 & \\
\hline
\end{tabular}

Table.2 Losses assessment of CLS of maize during Kharif 2018

\begin{tabular}{|c|c|c|c|c|c|}
\hline Replication & Treatment & Disease rating & PDI & $\begin{array}{c}\text { Yield( } \\
\text { gm/plot) }\end{array}$ & Yield loss (\%) \\
\hline \multirow[t]{2}{*}{ R1 } & Protected & 2.5 & $\begin{array}{c}14.28 \\
(22.20)\end{array}$ & 1186 & \multirow[t]{2}{*}{22.34} \\
\hline & Unprotected & 6.5 & $\begin{array}{c}62.22 \\
(52.07)\end{array}$ & 921 & \\
\hline \multirow[t]{2}{*}{$\mathbf{R 2}$} & Protected & 1.5 & $\begin{array}{c}17.14 \\
(24.46)\end{array}$ & 954 & \multirow[t]{2}{*}{24.00} \\
\hline & Unprotected & 6 & $\begin{array}{c}72.50 \\
(58.37)\end{array}$ & 725 & \\
\hline \multirow[t]{2}{*}{$\mathbf{R 3}$} & Protected & 2.0 & $\begin{array}{c}15.00 \\
(22.79)\end{array}$ & 891 & \multirow[t]{2}{*}{22.89} \\
\hline & Unprotected & 6.5 & $\begin{array}{c}71.25 \\
(57.58)\end{array}$ & 687 & \\
\hline \multirow[t]{2}{*}{ R4 } & Protected & 2.5 & $\begin{array}{c}21.66 \\
(27.74)\end{array}$ & 1127 & \multirow[t]{2}{*}{23.51} \\
\hline & Unprotected & 6 & $\begin{array}{c}81.25 \\
(64.34)\end{array}$ & 862 & \\
\hline \multirow[t]{2}{*}{ R5 } & Protected & 3 & $\begin{array}{c}21.25 \\
(27.45)\end{array}$ & 1052 & \multirow[t]{2}{*}{22.52} \\
\hline & Unprotected & 7.5 & $\begin{array}{l}79.33 \\
62.96)\end{array}$ & 815 & \\
\hline
\end{tabular}




\begin{tabular}{|c|c|c|c|c|c|}
\hline \multirow[t]{2}{*}{ R6 } & Protected & 2.5 & $\begin{array}{c}15.71 \\
(23.35)\end{array}$ & 926 & \multirow[t]{2}{*}{22.89} \\
\hline & Unprotected & 6 & $\begin{array}{c}87.69 \\
(69.46)\end{array}$ & 714 & \\
\hline \multirow[t]{2}{*}{ R7 } & Protected & 1.5 & $\begin{array}{c}18.00 \\
(25.10)\end{array}$ & 938 & \multirow[t]{2}{*}{25.58} \\
\hline & Unprotected & 6.5 & $\begin{array}{c}66.67 \\
(54.74)\end{array}$ & 698 & \\
\hline \multirow[t]{2}{*}{ R8 } & Protected & 3 & $\begin{array}{c}21.87 \\
(27.88)\end{array}$ & 877 & \multirow[t]{2}{*}{20.98} \\
\hline & Unprotected & 6 & $\begin{array}{c}72.94 \\
(56.65)\end{array}$ & 693 & \\
\hline \multirow[t]{3}{*}{ R9 } & Protected & 2 & 21.42 & 908 & \multirow[t]{3}{*}{18.94} \\
\hline & & & $(27.57)$ & & \\
\hline & Unprotected & 7 & $\begin{array}{c}80.71 \\
(63.95)\end{array}$ & 736 & \\
\hline \multirow[t]{2}{*}{ R10 } & Protected & 3.5 & $\begin{array}{c}12.85 \\
(21.01)\end{array}$ & 1225 & \multirow[t]{2}{*}{20.16} \\
\hline & Unprotected & 7 & $\begin{array}{c}76.15 \\
(60.77) \\
\end{array}$ & 978 & \\
\hline \multirow[t]{2}{*}{ R11 } & Protected & 3 & $\begin{array}{c}18.33 \\
(25.35)\end{array}$ & 945 & \multirow[t]{2}{*}{22.53} \\
\hline & Unprotected & 7.5 & $\begin{array}{c}77.4 \\
(61.61)\end{array}$ & 732 & \\
\hline \multirow[t]{2}{*}{ R12 } & Protected & 2 & $\begin{array}{c}17.22 \\
(24.52)\end{array}$ & 981 & \multirow[t]{2}{*}{24.36} \\
\hline & Unprotected & 6.5 & $\begin{array}{c}36.75 \\
(37.32)\end{array}$ & 742 & \\
\hline \multirow[t]{2}{*}{ R13 } & Protected & 3 & $\begin{array}{c}26.25 \\
(30.82)\end{array}$ & 832 & \multirow[t]{2}{*}{22.59} \\
\hline & Unprotected & 6 & $\begin{array}{c}82.94 \\
(65.60)\end{array}$ & 644 & \\
\hline \multirow[t]{2}{*}{ R14 } & Protected & 3.5 & $\begin{array}{c}11.42 \\
(19.75)\end{array}$ & 1025 & \multirow[t]{2}{*}{23.51} \\
\hline & Unprotected & 6 & $\begin{array}{c}81.25 \\
(64.34)\end{array}$ & 784 & \\
\hline \multirow[t]{2}{*}{ R15 } & Protected & 2 & $\begin{array}{c}18.75 \\
(25.66)\end{array}$ & 1124 & \multirow[t]{2}{*}{20.64} \\
\hline & Unprotected & 6.5 & $\begin{array}{c}70.00 \\
(56.79)\end{array}$ & 892 & \\
\hline \multirow[t]{2}{*}{ Mean } & Protected & 2.50 & $\begin{array}{c}18.08 \\
(25.04)\end{array}$ & 992.73 & \multirow{4}{*}{22.49} \\
\hline & Unprotected & 6.50 & $\begin{array}{c}73.27 \\
(\mathbf{5 9 . 2 4})\end{array}$ & 760.53 & \\
\hline \multicolumn{2}{|c|}{ T calculated } & 19.88 & $\begin{array}{c}17.77 \\
(17.33)\end{array}$ & 27.95 & \\
\hline \multicolumn{2}{|c|}{ T tabulated } & 1270 & 12.70 & 12.70 & \\
\hline
\end{tabular}


Int.J.Curr.Microbiol.App.Sci (2021) 10(09): 538-544

Table.3 Pooled data of losses assessment of Kharif 2017 and Kharif 2018

\begin{tabular}{|c|c|c|c|c|c|c|c|c|c|c|c|}
\hline \multirow[t]{2}{*}{ Treatments } & \multicolumn{3}{|c|}{ Disease rating } & \multicolumn{3}{|c|}{ Mean grain yield(gm) } & \multicolumn{3}{|c|}{ Mean PDI } & \multirow{2}{*}{\multicolumn{2}{|c|}{ Per cent yield loss }} \\
\hline & $\begin{array}{c}\text { Kharif } \\
2017\end{array}$ & $\begin{array}{c}\text { Kharif } \\
2018\end{array}$ & Pooled & $\begin{array}{c}\text { Kharif } \\
2017\end{array}$ & $\begin{array}{c}\text { Kharif } \\
2018\end{array}$ & Pooled & $\begin{array}{c}\text { Kharif } \\
2017\end{array}$ & $\begin{array}{c}\text { Kharif } \\
2018\end{array}$ & Pooled & & \\
\hline Protected & 2.33 & 2.5 & 2.41 & 1032.33 & 992.73 & 1012.53 & 18.56 & 18.07 & 18.31 & $\begin{array}{c}\text { Kharif } \\
2017\end{array}$ & $\begin{array}{c}\text { Kharif } \\
2018\end{array}$ \\
\hline Unprotected & 6.7 & 6.5 & 6.6 & 795.40 & 774.86 & 792.69 & 71.68 & 73.27 & 72.47 & 21.69 & 22.49 \\
\hline \multicolumn{3}{|c|}{ T calculated value } & 31.58 & & & $\begin{array}{c}34.94 \\
(33.97)\end{array}$ & & & 41.43 & \multicolumn{2}{|c|}{22.06} \\
\hline
\end{tabular}


During Kharif 2017(Table 4.10) the mean disease rating for protected plot were recorded with 2.33 and unprotected with 71.68 whereas, mean PDI for protected recorded $18.56 \%$ and $71.68 \%$ for unprotected plot.

Resulted yield in protected plot was 1032.33 $\mathrm{kg} / \mathrm{plot}$ and $795.40 \mathrm{~kg} / \mathrm{plot}$ in unprotected. The mean disease loss was recorded $21.69 \%$.

Similarly in Kharif 2018 (Table 4.11) mean disease rating was 2.5 and 6.5 in protected and unprotected plots respectively. Mean PDI and yield were recorded for protected was $18.07 \%$ and $992.73 \mathrm{~kg} / \mathrm{plot}$ and for unprotected $72.47 \%$ and $774.86 \mathrm{~kg} /$ plot respectively. Mean disease loss in Kharif 2018 was $22.49 \%$.

Pooled mean result of Kharif 2017 and kahrif 2018 (Table 4.12) for protected and unprotected is as follow the disease rating was 2.5 and 6.4, PDI was 18.31 and 72.47 and yield 1012.53 and 792.69 were observed. The mean yield lose of Kharif 2017 and kahrif 2018 was $22.06 \%$ recorded.

As per recent study the loss assessment of maize causes due to Curvularia leaf spot of maize the yield loss due to Curvularia leaf spot of maize was identified $22.06 \%$ that in epiphytic condition disease was cause economical dame to crop.

\section{References}

Akinbode, O. A. (2010). Evaluation of antifungal efficacy of some plant extracts on Curvularia lunata, the casual organism of maize leaf spot. African journal of Environmental science and Technology. 4(11): 797800.

Anonymous (2012). Faostat. Food and Agricultural Organization of the United Nations (FAO), FAO Statistical Database.

Cramer, H. H. 1967. Plant protection and world crop production. Bayer, Leverkusen, West Germany, Pp, 1-54.

Le Clerg, E. L. 1971. Field experiments for assessment of crop losses. In : Crop loss assessment methods, F.A.O. Manual on the evaluation and prevention of losses by pests, diseases and weeds (Ed. Chiarappa, L.). FAO and Commonwealth Agril. Bureaux, Great Britain, pp 1-11.

Macri, F. and P. Dilenna (1974). Com leaf blight incited by Curvularia lunata (Wall.) Baed. Rivisla di Palhologia Vegetable. 10: 27-35.

Prasanna, B., Vasal, S., Kassahun, B. and Singh, N. (2001). Quality protein maize. Current Science 81(10): 13081319.

\section{How to cite this article:}

Tarun Kumar Jatwa, S. S. Sharma, Neeraj K. Meena, Irfan Khan, Roop Singh and Indu. 2021. Evaluation of Yield Loss Assessment Caused due to Curvularia Leaf Spot (Curvularia lunata) in Maize. Int.J.Curr.Microbiol.App.Sci. 10(09): 538-544. doi: https://doi.org/10.20546/ijcmas.2021.1009.062 